\title{
COVID-19 drug repurposing: Summary statistics on current clinical trials and promising untested candidates
}

\author{
J. Ulm ${ }^{1}$ and Stanley Nelson ${ }^{1}$ \\ ${ }^{1} \mathrm{UCLA}$
}

April 27, 2020

\begin{abstract}
Repurposing of existing antiviral drugs, immunological modulators, and supportive therapies represents a promising path toward rapidly developing new control strategies to mitigate the devastating public health consequences of the COVID-19 pandemic. A comprehensive text-mining and manual curation approach was used to comb and summarize the most pertinent information from existing clinical trials. Drugs with previous efficacy against related betacoronaviruses like SARS and MERS were also systematically evaluated as potentially promising candidates for drug repositioning.
\end{abstract}

COVID-19 drug repurposing: Summary statistics on current clinical trials and promising untested candidates

Running Title: COVID-19 drug repurposing: Trials and prospects

J. Wes Ulm ${ }^{1,2,3}$ and Stanley F. Nelson ${ }^{1}$

${ }^{1}$ Department of Human Genetics, David Geffen School of Medicine, University of California, Los Angeles, Los Angeles, CA, USA

${ }^{2}$ Current address: Department of Medical Genetics, University of Pittsburgh School of Medicine, Pittsburgh, PA, USA

${ }^{3}$ Corresponding author

\section{Summary}

Repurposing of existing antiviral drugs, immunological modulators, and supportive therapies represents a promising path toward rapidly developing new control strategies to mitigate the devastating public health consequences of the COVID-19 pandemic. A comprehensive text-mining and manual curation approach was used to comb and summarize the most pertinent information from existing clinical trials and previous efforts to develop therapies against related betacoronaviruses, particularly SARS and MERS. In contrast to drugs in current trials, which have been derived overwhelmingly from studies on unrelated RNA viruses, a number of untested small molecule antivirals had previously demonstrated remarkable in vitro specificity for SARS-CoV or MERS-CoV, with high selectivity indices, $\mathrm{EC}_{50}$, and/or $\mathrm{IC}_{50}$. Due to the containment of the prior epidemics, however, these were largely not followed up with animal studies or clinical investigations and thus overlooked as treatment prospects in the current COVID-19 trials. This brief review summarizes and tabulates core information on the dozens of clinical trials currently in progress, while detailing the most promising untested candidates with prior documented success against the etiologic agents of SARS and/or MERS.

Keywords: COVID-19, drug repositioning, pandemics, antiviral agents, data mining, SARS 


\section{Main Text}

The unprecedented public health, economic, and social challenges engendered by the current COVID-19 pandemic necessitate an urgent search for effective clinical interventions to help reduce viral load and epidemiological spread, improve prevention and control, and stem the tide of rising morbidity and mortality (Spinelli \& Pellino, 2020). Due to the time lag of vaccine trials and de novo drug development based on standard drug-target modeling, compound screens, and multi-phase clinic testing, the most rapid and practical approach toward new clinical options lies in drug repositioning of proven or promising infectious control modalities (Li \& De Clercq, 2020). For SARS-CoV-2, the etiologic agent of COVID-19, this effort is significantly assisted by previous endeavors to develop therapeutics for two prior smaller epidemics, both caused by closely related coronavirus types. Severe acute respiratory syndrome (SARS) and Middle Eastern Respiratory Syndrome (MERS) each originated from outbreaks of betacoronaviruses with significant sequence and structural similarity to the SARS-CoV-2 betacoronavirus (Zhu et al., 2020).

Dozens of clinical trials have thus far been launched since January 2020, at multiple sites, to assay for efficacy of various repurposed drugs against SARS-CoV-2, most available on the National Library of Medicine's registry of clinical studies, others scraped from text-mining of the available literature. Figure 1 summarizes current trials in progress on the basis of the tested drugs, targets or mechanisms, preliminary efficacy studies, current and prior literature on the drug's efficacy for betacoronavirus-related disease, and the specific designating information for the relevant trials themselves including their phase, scale, expected completion date, and other key descriptors. This picture is rapidly changing as new efforts come online, but as of April 17, 2020, at least 53 separate public clinical trials were either in operation, completed with preliminary results, or in various stages of planning. The tested modalities attempt a variety of approaches to improve patient outcomes, some targeting the virus directly, others seeking to counter its deleterious physiological sequelae through immunomodulation or respiratory and circulatory support to reduce mortality and morbidity.

Summary statistics for these early trials are provided in Figure 2. As can be seen, drugs and targets linked to unique viral components and processes - such as the viral protease and RNA-dependent-RNA-polymerasepredominate in current trials. Several drugs have demonstrated early promise in at least some preliminary studies including lopinavir/ritonavir, remdesivir, and chloroquine or hydroxychloroquine, and more than a third (for which comprehensive descriptions were provided) are at or beyond Phase 3. Nevertheless, the scale of most trials remains small, the vast majority are not multi-centered, and evaluation of efficacy will take months or years to carry out. Only a few clinical trials are enrolling greater than 1,000 participants, including the global WHO DISCOVERY trial, the European-based INSERM trial, and the Adaptive COVID19 Treatment Trial. Moreover, the most commonly tested drugs have been drawn from antiviral studies outside the prior body of research focusing on SARS and MERS.

Since the SARS and MERS outbreaks dissipated without approaching the global impact of COVID-19, fledgling therapeutic studies for these epidemics were generally not followed up, and animal and human data are largely unavailable. However, a recent study noted significant in vitro and in vivo activity of a known nucleoside analog with previous efficacy against SARS and MERS, $\beta$-D-N4-hydroxycytidine (NHC), in reducing viral load in cell culture and tissue damage in mice secondary to SARS-CoV-2 infection (Sheahan et al., 2020). NHC was one of nearly a dozen drugs to have demonstrated potential in reducing the disease burden from SARS, MERS, or both (De Clercq, 2006; Kumar, Jung, \& Liang, 2013; Savarino, 2005), and the recent results support the notion that such repurposing may be fruitful for COVID-19.

We have therefore systematically combed available literature, reports, and commentaries to ascertain untested drugs with previous promise for SARS and MERS that merit consideration for additional COVID-19 trials, alongside the comprehensive clinical trial data elaborated previously. We utilized an approach combining careful manual curation and algorithmic scraping using a flexible Python language-based text-mining tool, previously developed for research into prospective repurposable drugs for Duchenne muscular dystrophy. Concomitantly, we systematically examined the drugs with previous reported efficacy in the context of SARS and MERS, then curated them on the basis of several factors most indicative of potential in clinical trials to repurpose them for SARS-CoV-2. 
In assessing criteria to identify highly promising candidates for COVID-19 drug repurposing, it has been noted that perhaps the primary predictor of eventual failure in clinical trials is non-selectivity for the target, contributing to unacceptable toxicity (Gayvert, Madhukar, \& Elemento, 2016). Therefore, in examining the as yet untested or seldom-tested SARS and MERS drugs with potential for COVID-19 repositioning, particular weighting was given to those exhibiting a low reported $\mathrm{EC}_{50}$ (or $\mathrm{IC}_{50}$ ) and high Selectivity Index (SI) from cell culture studies. Attention was likewise given to drugs which are not only selective for a viral target, especially a component indispensable for viral replication, but also substantially reduce viral load in vitro. Further consideration was given for modalities demonstrating confirmation of potential efficacy from multiple centers. With these factors as primary criteria for identifying COVID-19 drug repurposing candidates, several especially promising potential therapeutics were identified, summarized in Figure 3.

Of particular promise are a docking octapeptide, AVLQSGFR (Chou, Wei, \& Zhong, 2003; Gan et al., 2006), and a Phe-Phe dipeptide inhibitor, 18c (Shie et al., 2005), with marked selectivity (>1,000-fold) for the betacoronaviral protease of SARS-CoV (3C-like protease, also known as 3CL protease, 3CLpro, or Mpro) and demonstrated capacity to reduce viral load in cell culture. Both agents exhibit not only remarkable Selectivity Index (SI) values, but also low $\mathrm{IC}_{50}$ and/or $\mathrm{EC}_{50}$ measurements that suggest viability as practical drugs in vivo. As noted previously, the viral protease is also the most frequent target of COVID-19 clinical trials currently in progress or planning, but these candidates stand out for their proven ability to selectively target 3CLpro and to bring about a tangible reduction in viral infection capacity.

Encouraging results have also been observed for bananin (Huang, Zheng, \& Sun, 2008; Tanner et al., 2005; Wang et al., 2011), a viral helicase inhibitor with > 30-fold selectivity in cell culture studies; for calpain inhibitor VI (Barnard et al., 2004), with > 100-fold selectivity; and for the herbal extract hesperetin (Lin et al., 2005), with an SI $>300$ and a direct inhibitory effect on the 3CL protease. Bananin (Tanner et al., 2005) and calpain inhibitor VI (Barnard et al., 2004) have likewise been found to inhibit viral load and infection in vitro. Promising seminal studies for inhibition of SARS or MERS infection have also emerged for another protease inhibitor, cinanserin (Chen et al., 2005), and for nafamostat (Yamamoto et al., 2016), a cellular serine protease inhibitor that reduces viral spike protein priming. Alongside the recent findings by Sheahan and coworkers with NHC, such results suggest that animal studies and preliminary clinical trials with these agents, or closely related chemical derivatives, may prove fruitful in expanding the arsenal of drugs to combat the relentless spread, morbidity, and mortality of COVID-19.

\section{Acknowledgements}

The authors would like to acknowledge the valuable assistance of Florian Barthélémy, $\mathrm{PhD}$, in helping to format the figures and illustrations for this manuscript.

\section{Conflict of Interest Statement}

The authors certify that they have no affiliations with, or involvement in, any organization or entity with any financial interest (such as honoraria; educational grants; participation in speakers' bureaus; membership, employment, consultancies, stock ownership, or other form of equity interest; and expert testimony or patentlicensing arrangements), or non-financial interest (such as personal or professional relationships, affiliations, knowledge or beliefs) in the subject matter or materials discussed in this manuscript.

\section{Data Availability Statement}

Data sharing is not applicable to this article as no new data were created or analyzed in this study.

\section{Ethics Statement}

The authors confirm that the ethical policies of the journal, as noted on the journal's author guidelines page, have been adhered to. No ethical approval was required as this is a review article with no original research data.

Barnard, D. L., Hubbard, V. D., Burton, J., Smee, D. F., Morrey, J. D., Otto, M. J., \& Sidwell, R. W. (2004). Inhibition of severe acute respiratory syndrome-associated coronavirus (SARSCoV) by calpain inhibitors and 
beta-D-N4-hydroxycytidine. Antivir Chem Chemother, 15 (1), 15-22. doi:10.1177/095632020401500102

Chen, L., Gui, C., Luo, X., Yang, Q., Gunther, S., Scandella, E., . . Jiang, H. (2005). Cinanserin is an inhibitor of the 3C-like proteinase of severe acute respiratory syndrome coronavirus and strongly reduces virus replication in vitro. $J$ Virol, 79 (11), 7095-7103. doi:10.1128/jvi.79.11.7095-7103.2005

Chou, K. C., Wei, D. Q., \& Zhong, W. Z. (2003). Binding mechanism of coronavirus main proteinase with ligands and its implication to drug design against SARS. Biochem Biophys Res Commun, 308 (1), 148-151. doi:10.1016/s0006-291x(03)01342-1

De Clercq, E. (2006). Potential antivirals and antiviral strategies against SARS coronavirus infections. Expert Rev Anti Infect Ther, 4 (2), 291-302. doi:10.1586/14787210.4.2.291

Gan, Y. R., Huang, H., Huang, Y. D., Rao, C. M., Zhao, Y., Liu, J. S., . . Wei, D. Q. (2006). Synthesis and activity of an octapeptide inhibitor designed for SARS coronavirus main proteinase. Peptides, 27 (4), 622-625. doi:10.1016/j.peptides.2005.09.006

Gayvert, K. M., Madhukar, N. S., \& Elemento, O. (2016). A Data-Driven Approach to Predicting Successes and Failures of Clinical Trials. Cell Chem Biol, 23 (10), 1294-1301. doi:10.1016/j.chembiol.2016.07.023

Huang, J. D., Zheng, B. J., \& Sun, H. Z. (2008). Helicases as antiviral drug targets. Hong Kong Med J, 14 Suppl 4, 36-38.

Kumar, V., Jung, Y. S., \& Liang, P. H. (2013). Anti-SARS coronavirus agents: a patent review (2008 present). Expert Opin Ther Pat, 23 (10), 1337-1348. doi:10.1517/13543776.2013.823159

Li, G., \& De Clercq, E. (2020). Therapeutic options for the 2019 novel coronavirus (2019-nCoV). In Nat Rev Drug Discov (Vol. 19, pp. 149-150). England.

Lin, C. W., Tsai, F. J., Tsai, C. H., Lai, C. C., Wan, L., Ho, T. Y., . . . Chao, P. D. (2005). Anti-SARS coronavirus 3C-like protease effects of Isatis indigotica root and plant-derived phenolic compounds. Antiviral Res, 68 (1), 36-42. doi:10.1016/j.antiviral.2005.07.002

Savarino, A. (2005). Expanding the frontiers of existing antiviral drugs: possible effects of HIV-1 protease inhibitors against SARS and avian influenza. J Clin Virol, 34 (3), 170-178. doi:10.1016/j.jcv.2005.03.005

Sheahan, T. P., Sims, A. C., Zhou, S., Graham, R. L., Pruijssers, A. J., Agostini, M. L., . . Baric, R. S. (2020). An orally bioavailable broad-spectrum antiviral inhibits SARS-CoV-2 in human airway epithelial cell cultures and multiple coronaviruses in mice. Sci Transl Med . doi:10.1126/scitranslmed.abb5883

Shie, J. J., Fang, J. M., Kuo, T. H., Kuo, C. J., Liang, P. H., Huang, H. J., . . . Wong, C. H. (2005). Inhibition of the severe acute respiratory syndrome 3CL protease by peptidomimetic alpha,beta-unsaturated esters. Bioorg Med Chem, 13 (17), 5240-5252. doi:10.1016/j.bmc.2005.05.065

Spinelli, A., \& Pellino, G. (2020). COVID-19 pandemic: perspectives on an unfolding crisis. Br J Surg . doi:10.1002/bjs. 11627

Tanner, J. A., Zheng, B. J., Zhou, J., Watt, R. M., Jiang, J. Q., Wong, K. L., . . . Huang, J. D. (2005). The adamantane-derived bananins are potent inhibitors of the helicase activities and replication of SARS coronavirus. Chem Biol, 12 (3), 303-311. doi:10.1016/j.chembiol.2005.01.006

Wang, Z., Huang, J. D., Wong, K. L., Wang, P. G., Zhang, H. J., Tanner, J. A., . . Niccolai, N. (2011). On the mechanisms of bananin activity against severe acute respiratory syndrome coronavirus. Febs j, 278 (2), 383-389. doi:10.1111/j.1742-4658.2010.07961.x

Yamamoto, M., Matsuyama, S., Li, X., Takeda, M., Kawaguchi, Y., Inoue, J. I., \& Matsuda, Z. (2016). Identification of Nafamostat as a Potent Inhibitor of Middle East Respiratory Syndrome Coronavirus S Protein-Mediated Membrane Fusion Using the Split-Protein-Based Cell-Cell Fusion Assay. Antimicrob Agents Chemother, 60 (11), 6532-6539. doi:10.1128/aac.01043-16 
Zhu, N., Zhang, D., Wang, W., Li, X., Yang, B., Song, J., . . Tan, W. (2020). A Novel Coronavirus from Patients with Pneumonia in China, 2019.N Engl J Med, 382 (8), 727-733. doi:10.1056/NEJMoa2001017

\section{Figure legends}

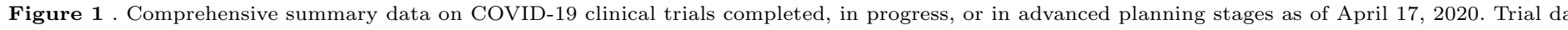

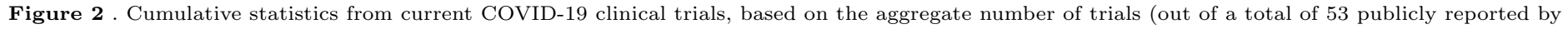

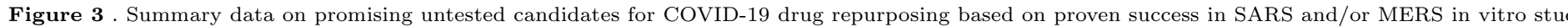

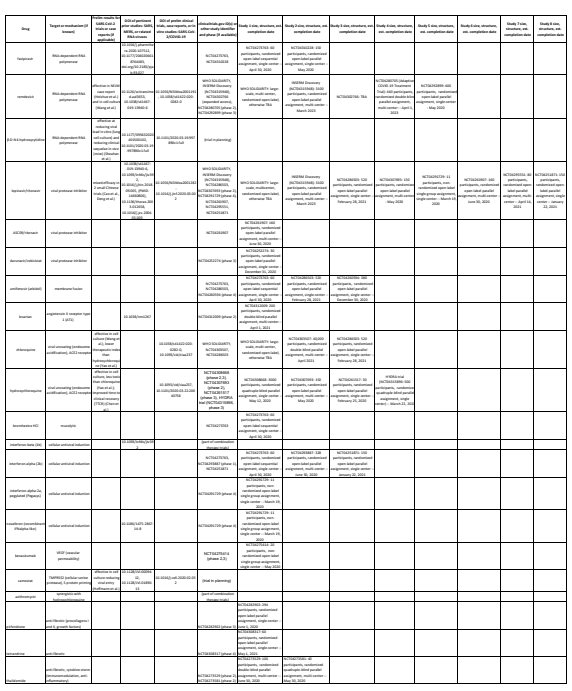


figures/COVID-19-drug-repurposing-JWU-SFN-Figure-2/COVID-19-drug-repurposing-JWU-SFN-Figu

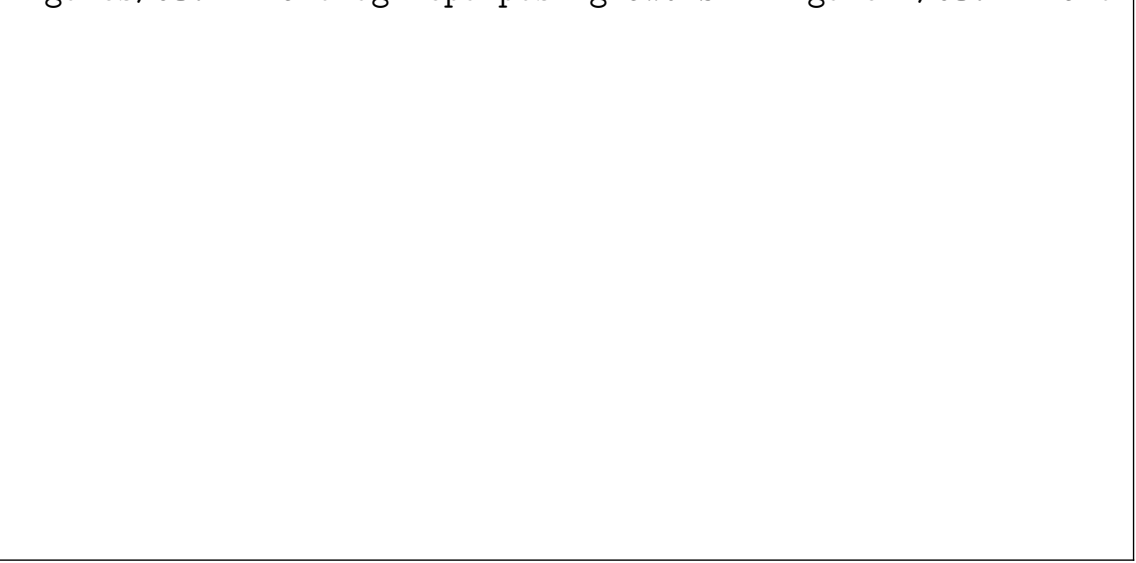


figures/COVID-19-drug-repurposing-JWU-SFN-Figure-3/COVID-19-drug-repurposing-JWU-SFN-Figu

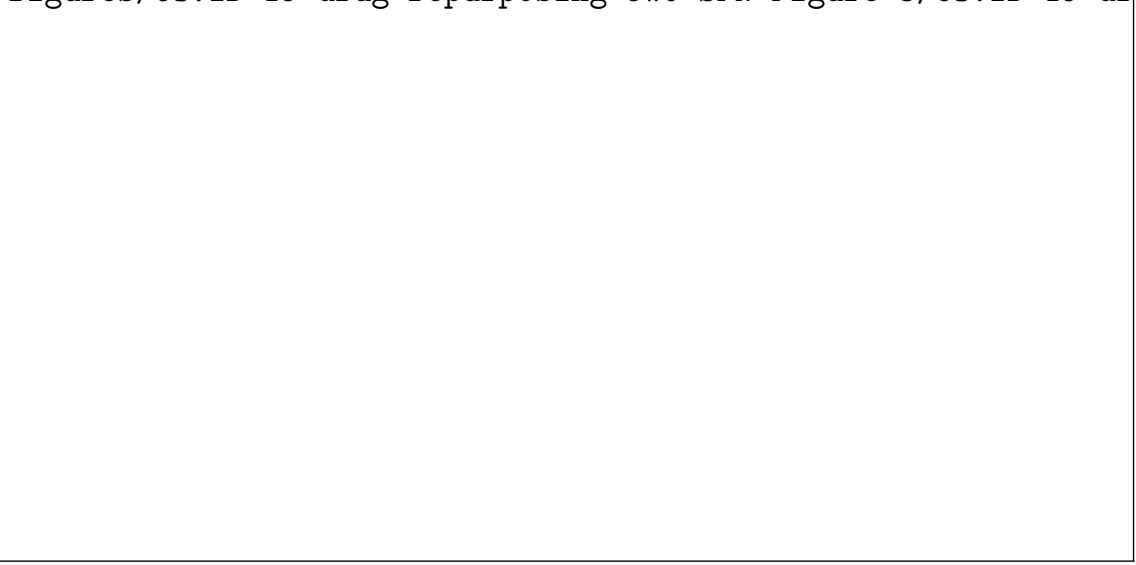

